\title{
A Novel Size-Selective Airborne Particle Size Fractionating Instrument for Health Risk Evaluation
}

\author{
B. GORBUNOV ${ }^{1}$, N. D. PRIEST ${ }^{2}$, R. B. MUIR ${ }^{1 *}$, P. R. JACKSON ${ }^{3}$ and \\ H. GNEWUCH
}

\author{
${ }^{1}$ Naneum Ltd, Suite BG10, CEH, University of Kent, Canterbury, Kent CT2 7NJ, UK; ${ }^{2}$ School of Health \\ and Social Sciences, Middlesex University, Queensway, Enfield, Middlesex EN3 4SA, UK; ${ }^{3}$ CERAM, \\ Queens Road, Penkhull, Stoke-on-Trent, Staffordshire ST4 7LQ, UK
}

Received 15 June 2008; in final form 19 December 2008; published online 11 March 2009

\begin{abstract}
Health risks associated with the inhalation of airborne particles are known to be influenced by particle size. Studies have shown that certain nanoparticles, with diameters $<100 \mathrm{~nm}$, have increased toxicity relative to larger particles of the same substance. A reliable, size-resolving sampler able to collect a wide range of particle sizes, including particles with sizes in the nanometre range, would be beneficial in investigating health risks associated with the inhalation of airborne particles. A review of current aerosol samplers used for size-resolved collection of airborne particles highlighted a number of limitations. These could be overcome by combining an inertial deposition impactor with a diffusion collector in a single device. Verified theories of diffusion and inertial deposition suggested an optimal design and operational regime. The instrument was designed for analysing mass distribution functions. Calibration was carried out using a number of recognized techniques. The sampler was tested in the field by collecting sizeresolved samples of lead containing aerosols present at workplaces in factories producing crystal glass. The mass deposited on each screen proved sufficient to be detected and measured by an appropriate analytical technique. Mass concentration distribution functions of lead were produced. The nanofraction of lead in air varied from 10 to $70 \%$ by weight of total lead.
\end{abstract}

Keywords: Brownian diffusion; humidity; inertial deposition; nanoparticles; size-resolved chemical composition; $\mathrm{PM}_{10}$

\section{INTRODUCTION}

The high health risk associated with the inhalation of airborne particles has been recognized and documented (see e.g. Brown et al., 2002; Pope et al., 1995). Many epidemiological studies have shown associations between exposure to particulate matter in the air and increases in morbidity and mortality, Dockery et al. (1993). There is a growing recognition that health risks associated with airborne particles are influenced by size. Some studies indicate that nanoparticles have increased specific toxicity relative to larger particles composed of the same materials (Donaldson et al., 1998; Ferin, 1994; Ferin et al., 1992; Oberdorster et al., 1995). Size-resolved sampling of the total aerosol is therefore necessary if health risks associated with inhalation of airborne particles are to be properly assessed.

*Author to whom correspondence should be addressed. Tel: +01227 824631; fax: +01227 827778;

e-mail: robert.muir@naneum.com
Size-resolved sampling of airborne particles requires various techniques to be employed (John, 2001). A significant benefit would therefore arise from a sampler that could reliably collect size-resolved samples across the entire size range which is considered to be relevant to health effects.

Cascade impactors have been employed for more than half a century to fractionate aerosol particles according to their aerodynamic diameter (Marple et al., 2001). Usually, a cascade impactor enables aerosol particles to be collected onto 5-10 successive impactor stages with decreasing cut-off diameters. For instance, in the May cascade impactor (May, $1945,1982)$, at $20 \mathrm{dm}^{3} \mathrm{~min}^{-1}$ flow rate, particles are separated into seven fractions (defined as diameters at which the collection efficiency is 50\%) with aerodynamic diameters ranging from $\sim 20$ to $0.25 \mu \mathrm{m}$. Other cascade impactors collect similar size ranges.

However, particles smaller than $\sim 0.25 \mu \mathrm{m}$ are also required to be size selectively collected to properly 
assess health risk. Since this is not possible using standard impactors, low-pressure cascade impactors have been developed to enable particles considerably $<0.25 \mu \mathrm{m}$ to be fractionated. Although low-pressure impactors can extend the range of size-selective sampling to particles with sizes of 20-30 nm, they cannot be accepted as ideal instruments for sampling particles in the nanosize range due to the following reasons: (i) The high pressure drop in low-pressure cascade impactors corrupts size distributions and causes condensation of water as well as other atmospheric constituents on substrates, e.g. Hart and Pankow (1994) have estimated that the gas-particle mass exchange for polycyclic aromatic hydrocarbons could cause errors in measurements of up to $40 \%$. Moreover, large mass changes were directly observed by Moor et al. (1998) in experiments where atmospheric aerosol particles collected onto substrates of a cascade impactor were exposed to conditions with lowered partial pressures of semi-volatile compounds. (ii) Low pressure may cause volatilization of some constituents and change the chemical composition of substances-especially organic substances. (iii) High flow velocity in a low-pressure impactor causes bouncing. Rotating substrates are used to increase the uniformity and to reduce bouncing. This increases the cost and weight of impactors. (iv) Impactors fractionate particles according to aerodynamic equivalent diameter. In nanosize ranges, deposition of particles in the respiratory tract depends primarily on diffusion. For this reason, mobility equivalent diameter is a better measure of nanoparticle sizes when evaluating health effects.

Therefore, there is a need for a wide-range aerosol sampling instrument that enables more reliable data on the size-resolved chemical composition of aerosols.

The operation of all cascade impactors is based upon the inertial deposition of aerosol particles.

Diffusivity, like inertial deposition, is influenced by the size of particles and can be used to derive size distributions. In the past, diffusion batteries were successfully employed to obtain aerosol particles size distributions in the nanoparticle range (e.g. Fuchs et al., 1962) - e.g. Sinclair and Hoopes (1975) developed the screen diffusion battery.

If a collector based on diffusion could be combined with a cascade impactor, many of the disadvantages of the low-pressure impactor could be overcome, providing a means for fractionating aerosol particles with a wide range of sizes.

In addition, human health risk is influenced by deposition of airborne particles at specific sites in the respiratory tract. The efficiency of deposition in the respiratory system was subject to experimental research and modelling, e.g. Chamberlain (1985), Hinds (1999), Lippmann (1995) and Yeh et al. (1996). It is well established that the deposition effi- ciency in the respiratory tract is influenced by the size of particles and forms a 'V-curve' comprising two branches caused by two main mechanisms of particle deposition: inertial deposition for larger particles with diameters $>200 \mathrm{~nm}$ and diffusion for smaller particles (diameter $<150 \mathrm{~nm}$ ) e.g. Wilson et al. (1985), Schiller et al. (1988), ICRP (1994) and Jaques and Kim (2000).

Using both diffusion and impactor collectors correctly accounts for both processes, without implication as to where in the respiratory tract deposition occurs.

The main advantage of such a combination is clearer in a comparison with data on nanoparticles collected using low-pressure cascade impactors. Deposition of nanoparticles in the respiratory tract is mainly controlled by diffusion and, therefore, calculation of the deposited dose from exposure requires knowledge of the diffusivity equivalent diameter Hinds (1999).

Some low-pressure cascade impactors are claimed to collect particles with sizes down to $20-30 \mathrm{~nm}$. However, low-pressure cascade impactors are based upon inertial deposition and, therefore, provide data on aerodynamic equivalent diameters. This creates uncertainties with interpretation of data in the nanorange particularly with respect to non-spherical particles of unknown density (e.g. agglomerates, fractals, soot particles, etc). Such particles are commonly present in aerosol samples.

It is quite difficult to calculate mobility equivalent diameters from aerodynamic equivalent diameters, even when the shape and density are known. For many practical cases, information regarding the shape and density is not available, making it very difficult to obtain robust information on deposition from this data. Uncertainties associated with this factor can cause significant errors in health risk evaluations.

However, combining a diffusion collector with an impactor requires a number of issues to be addressed.

In existing diffusion batteries, particles are collected onto nets. However, diffusion batteries such as these cannot be directly employed for sizeresolved sampling because they have a V-shaped dependence between collection efficiency and the size of particles (e.g. Kirsh and Stechkina, 1978). This means that there is not a one-to-one relationship between size and deposition efficiency. For example, the same collection efficiency corresponds to two different sizes, Fig. 1. Mass size distributions cannot, therefore, be easily retrieved from the mass deposited onto the substrates. (Note: this is not a problem when obtaining number concentration size distributions because fine and ultrafine particles are usually present in much greater numbers than coarse particles and the number of coarse particles can be neglected, but it is not possible to neglect the mass). 


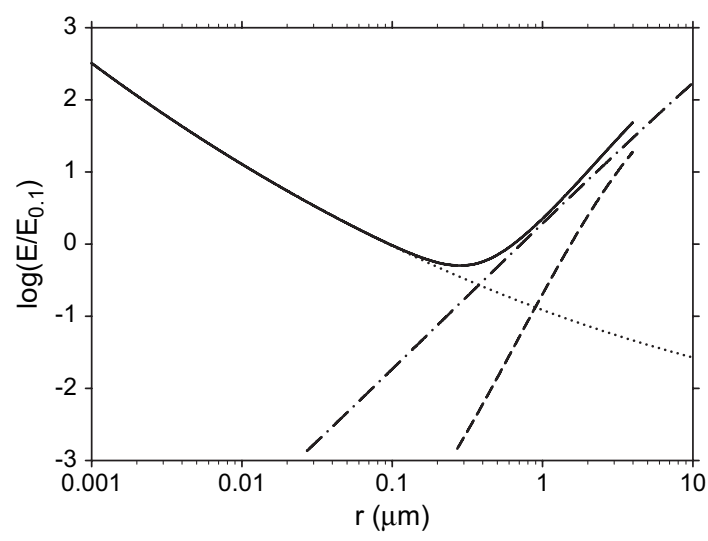

Fig. 1. A typical graph of the collection efficiency as a function of $r$ calculated for $T=293.15 \mathrm{~K}$, at atmospheric pressure $a=3.3 \times 10^{-5} \mathrm{~m}, Q=3.3 \times 10^{-5} \mathrm{~m}^{3} \mathrm{~s}^{-1}$, $d_{s}=4.7 \times 10^{-2} \mathrm{~m}, \alpha=0.7, \rho=1000 \mathrm{~kg} \mathrm{~m}^{-3}$,

$h=7.1 \times 10^{-5} \mathrm{~m}$ and $N=1$. The total collection efficiency, solid line; 1 , direct interception; 2 , inertial deposition; 3 , diffusion. The collection efficiency shown as a ration of $E(r) / E$ $(r=0.1 \mu \mathrm{m})$ in $\log$ scale.

In addition to the above, using existing diffusion batteries, like the TSI model 3040/3041, for mass analysis is difficult for practical reasons because of the great number of screens (55/56) and sections (10 covering two decades of sizes) deployed. For mass measurements, the mass of particles collected on a section needs to be sufficient to be detected by an appropriate analytical technique [usually atomic absorption spectroscopy (AAS), gas chromatography-mass spectrometry (MS) or inductively coupled plasma MS]. The diffusion particle collector should therefore be designed to maximize the mass deposited on screens by employing the smallest possible number of sections and screens.

Usually, diffusion batteries, such as those described in the literature or those commercially available, operate at flow rates of $\sim 5 \mathrm{dm}^{3} \mathrm{~min}^{-1}$ (e.g. Cheng et al., 1980) However, cascade impactors are often operated at higher flow rates of $\sim 20 \mathrm{dm}^{3} \mathrm{~min}^{-1}$ to minimize measurement errors, particularly if mass measurements are being made. In order to make a combined instrument, it is therefore desirable to develop a diffusion particle collector which operates at a flow rate that would make it compatible with high-flow-rate cascade impactors.

However, diffusion theory has only been developed for low flow rates (Fuchs, 1964; Kirsh and Stechkina, 1978; Cheng and Yeh, 1980) and it is not clear if it can be extended to high-flow-rate conditions. This is important because developing a diffusion unit without a theoretical foundation would be very difficult, especially when deposition kernels are required for data reduction. Obtaining size distributions with diffusion batteries is an 'ill-defined' problem that requires knowledge of kernel functions (e.g. Lesnic et al., 1995). Therefore, the performance of a diffusion collector working at $20 \mathrm{dm}^{3} \mathrm{~min}^{-1}$ has to be compared with theoretical predictions to be sure that the theory is still applicable and information obtained on particle sizes is robust.

Cascade impactors are often used to obtain size distributions without employing data inversion techniques. This approximation works well in many practical cases when size distributions are wider than the sharpness of the deposition efficiency on impactor stages. Developing a diffusion collector that also does not require data inversion techniques would have significant advantages in terms of ease of use. Understanding the resulting trade off between resolution and ease of use is therefore an important issue in developing a useful instrument.

Even if a diffusion collector has been developed with sufficient resolution, the question remains: Is the mass in the nanosize region sufficient to be detected using widely available techniques? The mass to number ratio rapidly decreases when reducing particle sizes by a factor of $10^{3}$ per decade. Consequently, between 1 and $100 \mathrm{~nm}$, the aerosol mass concentration is too small to be readily detected using most common techniques. Thus, it is necessary to demonstrate that enough mass is collected, within a reasonable time period, to allow mass determination.

In this paper, we address the following issues: (i) we discuss how a diffusion deposition collector can be designed and coupled with an inertial deposition unit within a single apparatus for the size-selective sampling of aerosol particles over a wide aerosol size range; (ii) we analyze if sufficient mass of particles can be accumulated in the nanosize region to be detected and (iii) we investigate if the diffusion deposition collector can be operated without data inversion techniques in a way similar to a cascade impactor.

\section{APPROACH}

Verified theories of diffusion deposition onto cylindrical fibres were used to determine the optimal geometry and regimes of the diffusion particle collector. A cascade impactor was designed to maximize simplicity and ease of use. The integrated instrument was calibrated using a number of standard techniques.

Laboratory and field tests were carried out to test the new instrument and to demonstrate situations where sufficient aerosol particle mass is present in the nanosize region, to be detected using conventional analytical techniques.

\section{CALCULATION OF THE DEPOSITION OF AEROSOL PARTICLES ONTO FIBRE NETS}

The diffusivity of aerosol particles decreases rapidly with increasing particle size (Fuchs, 1964). In the past, particle penetration measurements of an aerosol flowing through a diffusion element were 
employed to evaluate particle size distributions. The first diffusion batteries consisted of bundles of tubes or rectangular channels (Thomas, 1955; Fuchs et al., 1962; Sinclair, 1972). Sinclair and Hoopes (1975) developed a diffusion battery that consisted of layers of stainless steel screens. Diffusion batteries were applied for nanoparticles usually in the size range from 2 to $250 \mathrm{~nm}$ (e.g. Sinclair and Hoopes, 1975; Cheng et al., 1992).

The idea of using diffusivity for size-selective deposition can be illustrated with the conventional theory of aerosol particle deposition onto a screen or net containing cylindrical fibres (e.g. Kirsh and Stechkina, 1978; Cheng and Yeh, 1980). We consider the deposition of spherical aerosol particles with the density of $\rho=1000 \mathrm{~kg} \mathrm{~m}^{-3}$ onto a net comprising cylindrical infinite fibres positioned across a uniform flow of linear velocity $U$. The cross section of the flow is a circle of diameter $d_{s}$. According to the filtration theory of the wire screen diffusion battery, the deposition efficiency $E(r)$ of aerosol particles onto $N$ nets is

$$
E(r)=1-\exp \left[-\frac{2 N \alpha h}{\pi(1-\alpha) a} \eta(r)\right] .
$$

Here, $N$ is the number of nets, $\alpha$ is the solid volume fraction of the net that is normally calculated from $N, h$ and $a$, where $h$ is the net thickness $(M), a$ is the cylindrical fibre radius $(M), r$ [In theoretical calculations the radius $r$ is often used instead of the $(D)$ particles diameter to avoid confusion with the diffusion coefficient.] is the radius of the particles $(M)$ and $\eta(r)$ is the single wire collection efficiency. $\alpha$ can be calculated as the ratio of the volume of the fibres $V_{\mathrm{f}}$ to the volume of the net $V_{\mathrm{n}}, V_{\mathrm{n}}=h S$, where $S=\pi d_{s}^{2}$ and $S$ is the area of the net and $d_{s}$ is the net diameter. In practice, the volume of the fibres can be found from the weight of the net $W_{\mathrm{n}}$ and net material density $\rho=1130 \mathrm{~kg} \mathrm{~m}^{-3}\left(V_{\mathrm{f}}=W_{\mathrm{n}} / \rho\right)$. The single wire collection efficiency of aerosol particles can be described as a sum of deposition due to diffusion $\eta_{\mathrm{D}}(r)$, direct interception $\eta_{\mathrm{I}}(r)$, inertial impaction $\eta_{\mathrm{IN}}(r)$ and a correction term due to diffusion and interception $\eta_{\mathrm{DI}}(r)$ (Kirsh and Stechkina, 1978). The latter is relatively small and can be neglected, resulting in

$$
\eta(r)=\eta_{\mathrm{D}}(r)+\eta_{\mathrm{I}}(r)+\eta_{\mathrm{IN}}(r) .
$$

Hydrodynamic forces and electric forces play rather secondary roles in the deposition of aerosol particles onto fibres in the size range from $1 \mathrm{~nm}$ to $10 \mu \mathrm{m}$ (Fuchs, 1964; Kirsh and Stechkina, 1978). The diffusion term is calculated as follows according to Kirsh and Stechkina (1978) using the Peclet number Pe:

$$
\eta_{\mathrm{D}}(r)=2.7 / P e^{2 / 3}
$$

$P_{e}=2 a U / D_{r}$, where $U$ is the average fluid velocity across the net and $D_{r}$ is the diffusion coefficient of the particles,

$$
\begin{gathered}
U=4 Q /\left(\pi d_{s}^{2}\right) \\
D_{r}=k_{B} T C_{r} /\left(6 \pi m_{u} r\right)
\end{gathered}
$$

$k_{\mathrm{B}}$ here is the Boltzmann constant $(1.3806503 \times$ $\left.10^{-23} \mathrm{~m}^{2} \mathrm{~kg} \mathrm{~s}^{-2} \mathrm{~K}^{-1}\right), T$ is the temperature (K), $C_{r}$ is the slip correction factor;

$$
\begin{aligned}
m_{u} & =0.000184 p, \text { where } p \text { is the pressure } \mathrm{Nm}^{-2} \\
C_{r} & =1+1.246+0.42 \exp [-0.87 r / \lambda] \lambda / r .
\end{aligned}
$$

Here $Q$ is the flow rate, $m_{u}$ is the viscosity and $\lambda$ is the mean free path of the gas molecules in the air.

The term responsible for the direct interception was calculated according to equation (4), see Kirsh and Stechkina (1978):

$$
\eta_{\mathrm{I}}(r)=\frac{\left(1 /\left(1+R_{r}\right)\right)-1-R_{r}+2\left(1+R_{r}\right) \ln \left(1+R_{r}\right)}{2 c-\ln c-1.5-c^{2} / 2}
$$

Here $R_{r}=r / a$ and $c=2 \alpha / \pi$.

The inertial deposition term is expressed via the Stoke number Stk(r) (Kirsh and Stechkina, 1978)

$$
\begin{gathered}
\eta_{\mathrm{IN}}(r)=\operatorname{Stk}(r) \frac{\left[\left(29.6-28 \alpha^{0.62}\right) R_{r}^{2}-27.5 R_{r}^{2.8}\right]}{\sqrt{2 c-\ln c-1.5-0.5 c^{2}}} \\
\operatorname{Stk}(r)=\frac{\rho U r^{2} C_{r}^{2}}{9 m_{u} a}
\end{gathered}
$$

A numerical model based upon this theory has been developed to calculate the deposition of aerosol particles in a diffusion size-selective particle collector. The total deposition efficiency was calculated according to equations (1)-(5) for various flow rates and geometries.

A typical graph of the total deposition efficiency of aerosol particles onto a net positioned across a flow has a distinctive V-shape with a minimum at $r \sim$ $0.25 \mu \mathrm{m}(D \sim 0.5 \mu \mathrm{m})$ (Fig. 1). The curve contains two branches: the left branch $(r<0.25 \mu \mathrm{m})$ is mainly governed by diffusion while the right branch $(r>0.25 \mu \mathrm{m})$ is mainly influenced by inertial deposition and direct interception. The location of the minimum is influenced by the flow rate and the geometry as well as other properties of the diffusion collector but often the minimum is in the range from 0.1 to $0.7 \mu \mathrm{m}$ ). In the case of nets, the deposition efficiency versus size is not a monotonous function that corresponds to a one-to-one (size-efficiency) relationship.

Using nets allows the size-selective deposition only for aerosols of nanoparticles, when particles $>0.25 \mu \mathrm{m}$ are not present (Cheng et al., 1992). Nets cannot be used to sample aerosol particles across a wider range. However, there is one important 
feature of the curve in Fig. 1: the minimum is located at $\sim 0.25 \mu \mathrm{m}$, which is the size that is close to the lowest size stage of an ordinary cascade impactor. This gives the opportunity for combining a cascade impactor and a diffusion collector. The size-selective deposition of particles $<0.25 \mu \mathrm{m}$ is possible if larger particles are removed from the flow by a cascade impactor. After removing the larger particles from the flow, the subsequent collection of the rest of particles $(<0.25 \mu \mathrm{m})$ is governed by a monotonous function of collection efficiency versus size which enables aerosol particles to be selectively collected by nets.

\section{DESIGN OF THE WIDE-RANGE AEROSOL SAMPLER (WRAS)}

An elegant way to size selectively collect aerosol particles across a wide range of sizes from nanometres to micrometres would be to use a combination of a cascade impactor for larger particles and a diffusion unit to collect nanoparticles (Fig. 2). An example of such an instrument with a cascade impactor having the lowest stage cut-off diameter $0.25 \mu \mathrm{m}$ (May-type cascade impactor operating at $20 \mathrm{dm}^{3}$ $\mathrm{min}^{-1}$ ) is described. The numerical model presented in the previous section was employed to calculate the cut-off diameters. First, aerosol particles have to be drawn into the cascade impactor where particles $>0.25 \mu \mathrm{m}$ in diameter were collected. Collection efficiency of particles in an inertial cascade impactor increases with particle size. Therefore, the largest particles are removed from the flow within the first stage and the smaller particles are deposited onto the following stages.

The flow emerging out of the cascade impactor contains particles $<0.25 \mu \mathrm{m}$. These particles can be collected by a set of different nets. The collection efficiency of particles in a diffusion unit decreases with

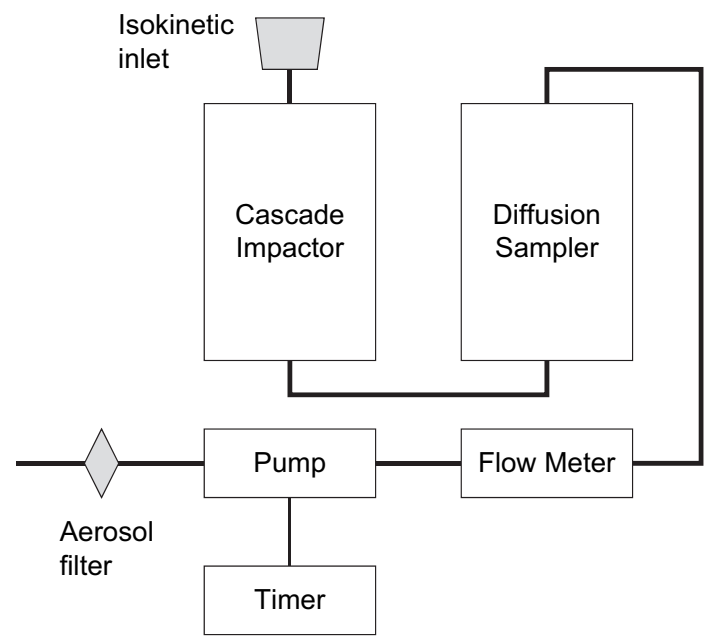

Fig. 2. Schematic of the wide-range aerosol sampling system WRAS. increased size. Therefore, the smallest particles are collected at the first net and the larger particles are deposited onto the following nets.

A diffusion unit has to be designed to collect sequentially particles in the size range from $1 \mathrm{~nm}$ to $0.25 \mu \mathrm{m}$. In practice, this is achieved by selecting nets with different fibre diameters and fibre densities, by varying the number of nets per stage and by choosing the appropriate flow velocity. In principle, the unit is quite similar to the design of a cascade impactor. Our diffusion collection unit has a cylindrical cross section (ID $=4.7 \mathrm{~cm}$ and $\mathrm{OD}=6.7 \mathrm{~cm}$ ) and contains five stages (Fig. 3). The unit was made from aluminium and black rubber o-rings are used to separate the stages. Each stage contains either one or several nylon nets.

Deposition efficiencies for the diffusion aerosol particle collector are quite different than efficiencies for cascade impactors because the diffusion collection efficiency of a diffusion collector $E(D)$ decreases with increasing particle size-see Fig. 1 as an example. Penetration efficiencies $P(D)$ that are equal to

$$
P(D)=1-E(D)
$$

are similar to the collection efficiencies of an inertial instrument.

To demonstrate the mode of action, calculations of penetration efficiency were performed for a collector with four stages. The results are shown in Fig. 4 and Table 1. In this figure, curve 1 corresponds to a cutoff diameter of $3.1 \mathrm{~nm}$, i.e. $P(3.1)=50 \%$. Thus, particles $<3.1 \mathrm{~nm}$ will be captured by the first net and

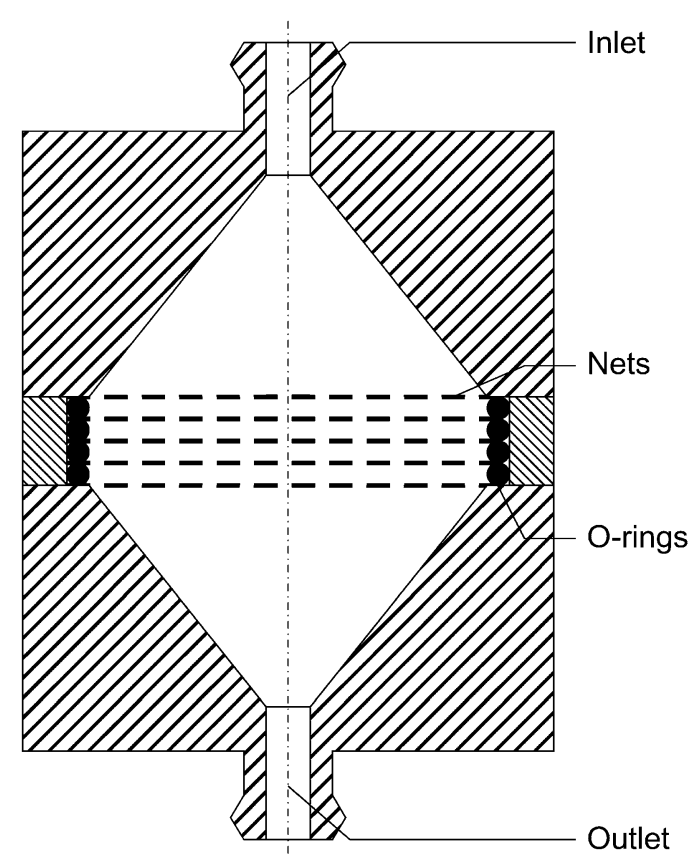

Fig. 3. Schematic of the diffusion particle collector. 
larger particles will be carried to the next stages. In the second stage, curve 2, particles are collected in the size range from 3.1 to $12 \mathrm{~nm}$. Larger particles deposit on stages 3-5.

The curves describing the performance of the diffusion unit are similar to those for the cascade impactor. In general, they are smoother than curves for inertial deposition. This is because the collection efficiency in the inertial region $(r>0.25$ or $D>0.5$ $\mu \mathrm{m}$, Fig. 1) is a steeper function than in the diffusion region $(D<0.5 \mu \mathrm{m})$.

\section{THE CHOICE OF NETS}

In a conventional diffusion battery, the same type of screen/net (in terms of mesh size, threads per $\mathrm{mm}$, etc.) is usually used for different stages. In the diffusion size-selective particle collector, different types of nets are required to cover the whole size range with cut-off diameters distributed more or less

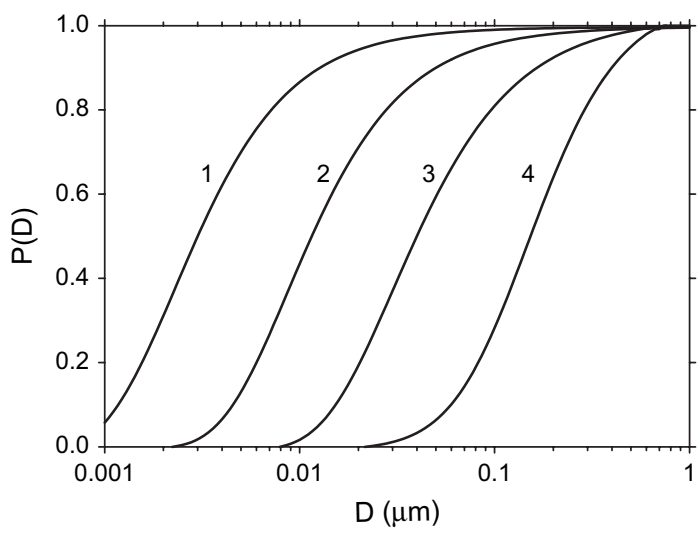

Fig. 4. An example of penetration efficiencies for the diffusion collector with four stages. Calculated at $T=293.15 \mathrm{~K}$, $p=101325 \mathrm{~Pa}, Q=1.7 \times 10^{-4} \mathrm{~m}^{3} \mathrm{~s}^{-1}, d_{s}=4.7 \times 10^{-2} \mathrm{~m}$. For the first stage: $\alpha_{1}=0.32, N_{1}=1$ and $a_{1}=4.2 \times 10^{-5} \mathrm{~m}$; for the second stage: $\alpha_{2}=0.43, N_{2}=2$ and $a_{2}=1.7 \times$

$10^{-5} \mathrm{~m}$; for the third stage: $\alpha_{3}=0.6, N_{3}=5$ and

$a_{3}=1.7 \times 10^{-5} \mathrm{~m}$; for the fourth stage: $\alpha_{4}=0.7$, $N_{4}=20$ and $a_{4}=1.5 \times 10^{-3} \mathrm{~m}$.

Table 1. Cut-off diameters ${ }^{\mathrm{a}} D_{i}$ [corresponding $P\left(D_{i}\right)=50 \%$ ] of the size-selective diffusion collector with four stages

\begin{tabular}{lc}
\hline Stage & $D_{i}, \mathrm{~nm}$ \\
\hline 1 & 3.1 \\
2 & 12 \\
3 & 40 \\
4 & 150 \\
\hline
\end{tabular}

$P\left(D_{i}\right)$ values were calculated according to equations (1)-(5); details are shown in the captions to Fig. 4.

${ }^{\mathrm{a}}$ Cut-off diameters $D_{i}$ for penetration and for deposition efficiencies are equal according to equation (6). It is possible to use upper cut-off diameters for the deposition efficiency. equally (Cheng et al., 1992). In practice, there is a limited number of nets available. Nylon net filters (Millipore) with various mesh openings (from 11 to $180 \mu \mathrm{m}$ ) were used in the diffusion size-selective particle collector because of their relatively low cost and their compatibility with AAS and other analytical techniques. The nets were chosen following numerical modelling and after confirmatory calibration.

It is important to use different types of net filters. It was found that using the same type of nets for all stages meant the number of nets would be very large, making their use very expensive and impractical (assembly and analysis). For instance, using a net type with $180 \mu \mathrm{m}$ mesh opening requires almost 100 net filters to catch aerosol particles of $0.25 \mu \mathrm{m}$ diameter.

\section{THE IMPORTANCE OF THE INTERFACE BETWEEN A DIFFUSION COLLECTOR AND A CASCADE IMPACTOR}

In the wide-range aerosol sampler (WRAS), a sizeselective diffusion particle collector and a cascade impactor have to be designed to perform as a single unit, preferably with the same flow rate. This can be achieved if the cut-off sizes of both parts correspond to a single set of size sections without large gaps or overlapping in the deposition range of sections. The correct interface of both parts, which is based upon different mechanisms of deposition, is important because the size-selective diffusion particle collector collects smaller particles first but the cascade impactor, on the contrary, collects larger particle first. The reverse order of the particle collection is the main problem. The cascade impactor lowest stage cut-off size should be equal to the highest section diffusion collector cut-off size. If these cut-off sizes are not equal, then the reconstruction of the size distribution from the masses measured will be complicated. In practice, it can cause difficulties of interpretation because of possible overlapping cut-off sections or large gaps.

Figure 5 shows how a correct interface can be achieved for an instrument with a May-type cascade impactor. This version of the WRAS instrument has 11 stages including five stages for the diffusion aerosol particle collector. The cut-off diameters for the cascade impactor were taken from a certified commercial instrument. The cut-off diameters for the diffusion collector were calculated for $20 \mathrm{dm}^{3} \mathrm{~min}^{-1}$ and $d_{s}=4.3 \times 10^{-2} \mathrm{~m}$. The cut-off diameter of the fifth diffusion collector stage (the dashed line in Fig. 5) $0.24 \mu \mathrm{m}$ is very close to the cut of diameter of the fifth stage of the cascade impactor -0.25 $\mu \mathrm{m}$, see Table 2 .

The dashed curve in Fig. 5 corresponds to $5^{*}$ stage.

The cut-off sizes for stages 5 and $5^{*}$ are very close, but the shapes of the curves are slightly different. It is almost impossible to achieve the ideal fit of these 


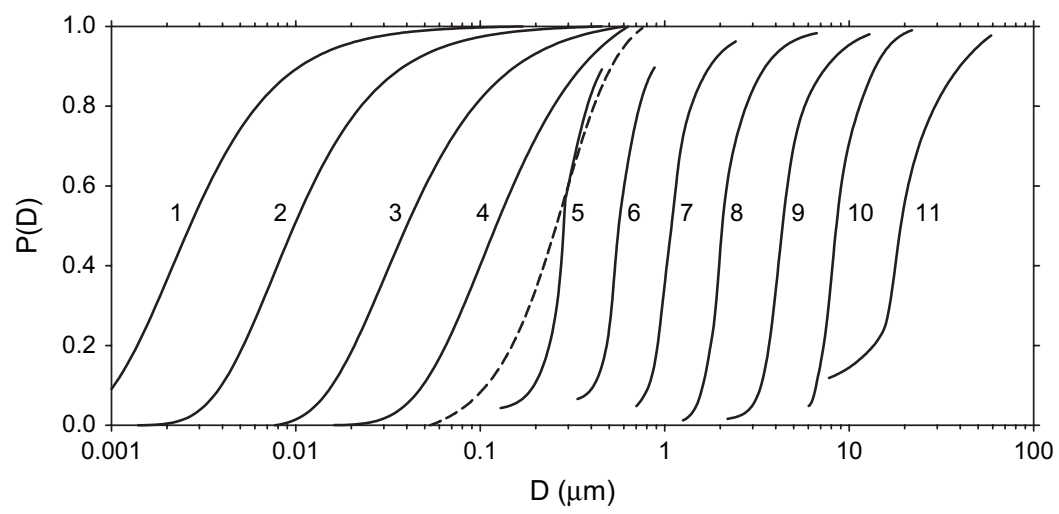

Fig. 5. Efficiencies for the wide-range aerosol sampling system. Curves 1-4 and the dashed line are penetration efficiencies of aerosol particles in the diffusion collector. Curves 5 to 11 are deposition efficiencies of aerosol particles in the May cascade impactor. $T=293 \mathrm{~K}, p=101325 \mathrm{~Pa}, Q=3.3 \times 10^{-4} \mathrm{~m}^{3} \mathrm{~s}^{-1}$ and $d_{s}=4.7 \times 10^{-2} \mathrm{~m}$.

Table 2. The characteristics of the stages of the wide-range aerosol sampler (WRAS)

\begin{tabular}{cccccr}
\hline Stage & $\begin{array}{l}\text { Minimal } \\
\text { cut-off } \\
\text { diameter } \\
D_{i}, \mu \mathrm{m}\end{array}$ & $\begin{array}{l}\text { Maximum } \\
\text { cut-off } \\
\text { diameter } \\
D_{i}, \mu \mathrm{m}\end{array}$ & $a, \mathrm{~cm}$ & $\alpha$ & $N_{i}$ \\
\hline 1 & 0.001 & 0.0015 & 0.0042 & 0.32 & 1 \\
2 & 0.0015 & 0.005 & 0.0017 & 0.6 & 1 \\
3 & 0.005 & 0.015 & 0.0017 & 0.6 & 5 \\
4 & 0.015 & 0.06 & 0.0015 & 0.78 & 12 \\
$5^{*}$ & 0.06 & 0.24 & 0.0008 & 0.81 & 50 \\
5 & 0.25 & 0.25 & & & \\
6 & 0.5 & 0.5 & & & \\
7 & 1.0 & 1.0 & & & \\
8 & 2.0 & 2.0 & & & \\
9 & 4.0 & 4.0 & & & \\
10 & 8.1 & 8.1 & & & \\
11 & 20 & 20 & & & \\
\hline
\end{tabular}

curves because of differences in the mechanism of the deposition.

This way of collecting ultrafine particles does not require low pressure. The total pressure drop across the impactor and the diffusion particle collector was $<6 \times 10^{3} \mathrm{~Pa}$. Thus, the particles deposited were exposed at a pressure close to atmospheric pressure.

The quality of an interface can be tested in experiments measuring the total penetration of aerosol particles through the WRAS instrument. As an example, for an imperfect interface between the diffusion and impaction based unit, we assume that the highest (diffusion) cut-off size is lower than the lowest (impaction) cut-off size. For this case, some particles will not be collected by WRAS. These particles, therefore, can be captured by an aerosol filter placed between the diffusion collector and the pump.

The total penetration of a WRAS instrument has been evaluated in experiments with atmospheric aerosol particles $0.2 \mu \mathrm{m}$ median diameter $\left(\sigma_{g}=1.6\right.$ and $N=3 \times 10^{4} \mathrm{~cm}^{-3}$ ) using a condensation particle counter. It was found that the aerosol particle number concentration measured at the exit of the diffusion collector was in the range from 35 to $50 \mathrm{~cm}^{-3}$ (24 measurements). The penetration was therefore $<0.2 \%$. This verifies that all the particles are collected and that there are no gaps in the size sections between the cascade impactor and the diffusion collector.

Reineking and Porstendorfer (1990) and Cheng et al. (1992) employed diffusion batteries for the collection of radioactive particles in order to obtain activity size distributions. These instruments have not been used to collect aerosol particles for mass analysis mainly because they have been designed to measure activity and the specific requirements for the mass analysis, discussed in the Introduction, have not been met.

\section{RESOLUTION AND RECONSTRUCTION OF THE SIZE DISTRIBUTION}

The slope of curves corresponding to the diffusion collector is not as steep as for the inertial deposition (Fig. 5). Hence, the resolution of the diffusion collector is lower than the resolution of the cascade impactor. The particle size distribution in a WRAS sampler can be calculated from a given set of deposition or penetration data.

The conventional diffusion battery has the same problem. Various methods have been suggested to reconstruct the size distribution from diffusion battery data. Some methods require the assumption of a lognormal distribution function (Fuchs et al., 1962; Mercer and Greene, 1974). The non-linear iterative inversion algorithm was suggested by Twomey (1975) for the reduction of diffusion battery penetration data. Thomas (1955) described a procedure by which the fraction larger or smaller than a given size can be calculated. Theoretical analysis enables size 
distributions to be retrieved using different mathematical schemes developed, e.g. by Soderholm (1979), Busigin et al. (1980), Lee et al. (1981), Maher and Laird (1985) and Lesnic et al. (1995). The graphical stripping technique has been described by Sinclair (1972). These methods have been applied for both the screen battery and a collimated-holes battery (Sinclair and Hoopes, 1975).

In general, deconvolution methods are usually employed to determine the size distribution of aerosols after collection in diffusion devices. For example, the TSI Diffusion Battery (Model 3040/3041) requires such a technique because it has 10 stages of classification and 11 sampling ports. These methods often require special software. They are also tedious and considerable operator experience is needed. In contrast, the WRAS instrument does not normally require the application of such methods and if they were used, in most cases, the same result would be obtained using deconvolution as would be obtained without it.

For WRAS, deconvolution may be used to increase the precision of measurements only when the size distribution of particles within an aerosol is narrowe.g. for monodispersed aerosols. In most cases, high resolution is not necessary. Such problems can be solved with two or even fewer stages per decade of size. In the WRAS instrument, five nanorange stages are most often deployed to collect particles in the size range from $1 \mathrm{~nm}$ to $0.25 \mu \mathrm{m}$. With five stages, considerable distortion of size distributions only occurs if they are narrow functions or monodisperse. At working places normally size distributions are wide (see Figs 6-8), therefore, distortions can be assumed to be small. Therefore, the diffusion size-selective particle collector can be used exactly as a cascade impactor without deconvolution of the data obtained. This should be considered as a first approximation of the size distribution evaluation. This compares well with the function of a cascade impactor which is also not a sharp step function. Retrieving narrow size distributions using a cascade impactor also requires some deconvolution of the data.

\section{Approximation of the wide size distribution}

In the WRAS instrument described above, the aerosol particle mass size distribution $f\left(D_{i}\right)$ can be found according to

$$
f\left(D_{i}\right)=\frac{\Delta m_{i}}{\log D_{i}-\log D_{i-1}},
$$

where masses $\Delta m_{i}$ is the mass found at $i$ th stage $(1 \leq$ $i \leq 5$ ). The same approach is usually used for cascade impactors.

\section{Approximation of the narrow size distribution}

When aerosol size distributions are narrow or if more accurate size distributions are required, a simple correction method can be used. The method is based

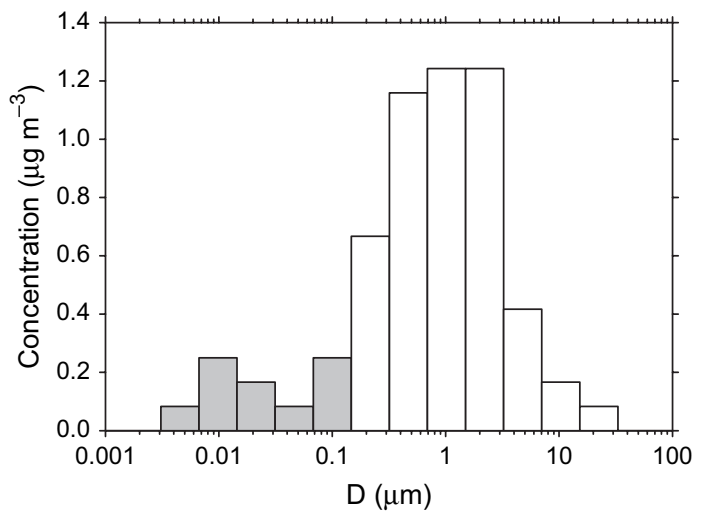

Fig. 6. Harmonized lead mass aerosol size distribution (dm/ $\mathrm{d} \log \mathrm{D}$ ) obtained at a glass factory processing lead.

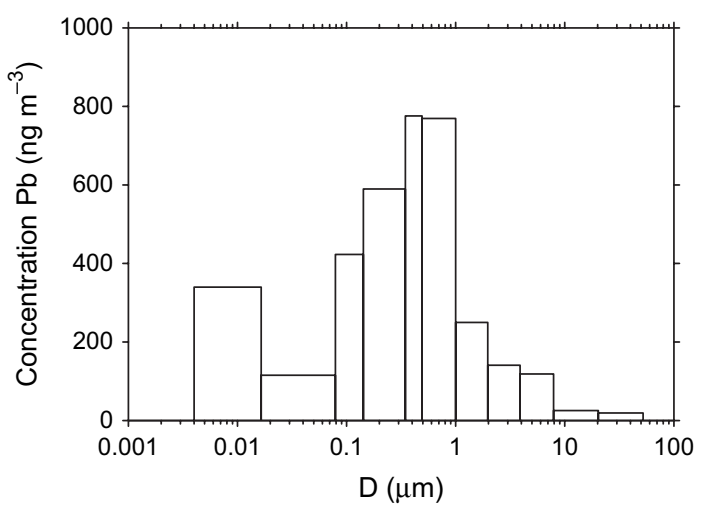

Fig. 7. Airborne lead particle size distribution obtained at a plant A involved in hot lead processing.

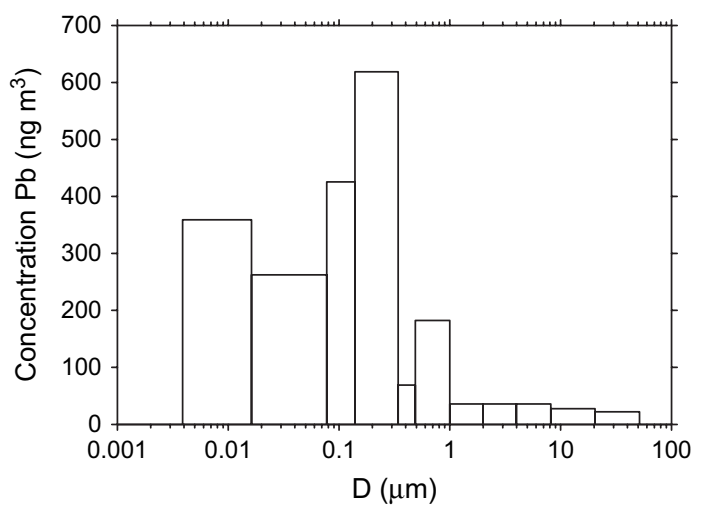

Fig. 8. Airborne lead particle size distribution obtained at a plant B involved into hot lead processing.

upon taking into account overlaps in the $P(D)$ functions (Fig. 5). It is assumed that the aerosol mass $\Delta m_{i}$ collected on stage $i$ is distorted by overlapping with that collected on the two nearest neighbour stages: $(i-1)$ th and $(i+1)$ th stages. The measured $M_{i}$ mass is a superposition of the set of the true masses $\Delta m_{j}$ and the deposition functions $a_{i j}$. For 
instance, for the first stage, the mass conservation law gives

$$
M_{1}=a_{11} \Delta m_{1}+a_{12} \Delta m_{2} .
$$

Here $a_{11}$ and $a_{12}$ are determined by $P_{1}(D)$

$$
\begin{aligned}
& a_{11}=\int_{0}^{\log D_{1}}\left[1-P_{1}(D)\right] \mathrm{d} \log D ; \\
& a_{12}=\int_{\log D_{1}}^{\log D_{2}}\left[1-P_{1}(D)\right] \mathrm{d} \log D .
\end{aligned}
$$

Here $P_{1}(D)$ is the penetration function $P(D)$ for stage 1 . It was accepted that particles $<1 \mathrm{~nm}$ diameter could not be collected by the first stage. This assumption is not very important for the size distribution because it affects only the lowest stage. A similar assumption is usually made for the first and the last stages of a cascade impactor.

Equation (8) shows that the mass collected by the net of the first stage is the sum of the mass fraction in the size section ( 1 to $D_{1}$ ) determined by $a_{11}$ and the mass in the size section $\left(D_{1}-D_{2}\right)$ determined by $a_{12}$. Therefore, some mass fraction deposited onto the first net actually belongs to the second interval: from $D_{1}$ to $D_{2}$ due to smoothness of the penetration efficiencies (Fig. 5).

The second stage collection performance is determined by equation (10) that is similar to equation (8)

$$
M_{2}=a_{21} \Delta m_{1}+a_{22} \Delta m_{2}+a_{23} \Delta m_{3} .
$$

The collection performance of the rest of the stages is described by equations that contain $a_{i j}$ and $\Delta m_{j}$. The $a_{i j}$ coefficients can be arranged as a matrix $\mathbf{A}$

$$
A=\left[\begin{array}{lllll}
a_{11} & a_{12} & 0 & 0 & 0 \\
a_{21} & a_{22} & a_{23} & 0 & 0 \\
0 & a_{32} & a_{33} & a_{34} & 0 \\
0 & 0 & a_{43} & a_{44} & a_{45} \\
0 & 0 & 0 & a_{54} & a_{55}
\end{array}\right] .
$$

Here $a_{i i}$ is defined as follows for $1<i \leq 5$

$$
a_{i i}=\int_{\log D_{i-1}}^{\log D_{i}}\left[P_{i-1}(D)-P_{i}(D)\right] \mathrm{d} \log D
$$

Here $P_{i}(D)$ is the penetration function $P(D)$ for stage $i$. Matrix elements $a_{i, i+1}$ and $a_{i, i-1}$ are defined by equation (13) that are similar to equations (9) and (12)

$$
\begin{aligned}
& a_{i, i+1}=\int_{\log D_{i}}^{\log D_{i+1}}\left[P_{i-1}(D)-P_{i}(D)\right] \mathrm{d} \log D(1 \leq i \leq 4) \\
& a_{i, i-1}=\int_{\log D_{i-2}}^{\log D_{i-1}}\left[P_{i-1}(D)-P_{i}(D)\right] \mathrm{d} \log D(2 \leq i \leq 5) .
\end{aligned}
$$

The values $a_{i j}$ have to be calculated only once for a particular type of WRAS instrument. Being calculated, they enable the true mass to be found, using simple numerical methods, for instance using linear algebra matrix methods. Let us construct the set of the true mass $\Delta m_{i}$ as a vector $\mathbf{m}_{\mathrm{x}}$. This vector is linked to the vector of measured mass $\mathbf{m}_{\mathrm{m}}$ by the linear equation according to equation (14)

$$
\mathbf{A} \times \mathbf{m}_{\mathrm{X}}=\mathbf{m}_{\mathrm{m}}
$$

The vectors $\mathbf{m}_{\mathrm{x}}$ and $\mathbf{m}_{\mathrm{m}}$ are defined as follows

$$
m_{x}=\left[\begin{array}{l}
\Delta m_{1} \\
\Delta m_{2} \\
\Delta m_{3} \\
\Delta m_{4} \\
\Delta m_{5}
\end{array}\right] m_{m}=\left[\begin{array}{l}
M_{1} \\
M_{2} \\
M_{3} \\
M_{4} \\
M_{5}
\end{array}\right]
$$

The solution of this linear equation is

$$
\mathbf{m}_{\mathrm{x}}=\left[\Delta m_{i}\right]=\left[\frac{\operatorname{Det} A_{i}}{\operatorname{Det} A}\right]
$$

Here $\operatorname{Det} A$ is the determinant $\mathbf{A}$ and $\operatorname{Det} A_{i}$ is the determinant obtained from $\operatorname{Det} A$ by replacing in $\operatorname{Det} A$ the $i$ th column by the column with the entries $M_{1}, \ldots M_{5}$. Alternatively, equation (14) can be solved by Gauss Elimination or another standard method for solving linear systems, which are widely available.

Matrix [equation (11)] that determines the performance of the diffusion collector (version of the WRAS instrument described above) is

$$
\left[\begin{array}{lllll}
0.74 & 0.26 & 0 & 0 & 0 \\
0.28 & 0.51 & 0.21 & 0 & 0 \\
0 & 0.22 & 0.54 & 0.24 & 0 \\
0 & 0 & 0.19 & 0.61 & 0.2 \\
0 & 0 & 0 & 0.30 & 0.7
\end{array}\right] .
$$

This approach enables aerosol particle size distributions to be corrected in the case of overlapping functions that describe the performance of the stages. It does not require complex calculations and can be easily implemented using standard software, for instance in MS Excel.

Thomas (1955) described a procedure by which the fraction of particles larger or smaller than a given size can be found. In our method, we calculate the true distribution assuming that the overlapping in the deposition functions affects only neighbouring stages.

\section{RESULTS}

\section{Calibration of the WRAS instrument}

Diffusion batteries have been calibrated extensively in the past (Sinclair and Hoopes, 1975; Cheng et al. 1980; Mavliev et al. 1984). Sinclair et al. (1976) have calibrated the screen battery with monodispersed aerosols. The models describing deposition of aerosol particles in the diffusion batteries also 
have been verified (e.g. Fuchs et al., 1962; Cheng et al., 1980). Sinclair and Hoopes (1975) have found the relationship between the screen battery and a collimated-holes battery.

In this paper, atmospheric aerosols and tungsten (W) aerosols were used to verify the performance of the diffusion collector. Artificial W aerosols were generated with a calibrated photochemical standard aerosol generator (Dubtsov and Baklanov, 1996).

The mass of $\mathrm{Pb}$ and $\mathrm{W}$ collected was determined by AAS with graphite furnace. A Perkin Elmer 1100 B spectrometer was employed. Aerosol particles with an aerodynamic diameter $>0.25 \mu \mathrm{m}$ were collected onto glass slides. Smaller particles were captured by Nylon filter nets of the nanoparticle collector. All substrates collected were carefully treated with aqua regia and subjected to a microwave digestion in sealed Teflon vessels. The samples obtained were then mixed with a catalyst and placed on a graphite platform within the furnace. Different temperature/time profiles were followed to drive off the water and ultimately bring about the volatilization process required for the absorbance measurement.

The lead mass was measured with $\mathrm{PM}_{2.5}$ occupational health $(\mathrm{OH})$ samplers (Casella, UK) and with the WRAS instrument $\left(\mathrm{PM}_{2.5}\right)$. Regression analysis on six samplers shows $R^{2}=0.82$ and the mean ratio of OH/WRAS $=1.1$. Thus, both instruments enable consistent data to be obtained.

Artificial W aerosols were obtained of the mean diameter $21 \mathrm{~nm} \sigma_{g}=1.4$ (concentration: $N=3.3 \times$ $\left.10^{5} \mathrm{~cm}^{3}\right)$ and $75 \mathrm{~nm} \sigma_{g}=2.3\left(N=5.2 \times 10^{4}\right.$ $\mathrm{cm}^{3}$ ). The size distribution of $\mathrm{W}$ aerosol particles was determined with an Scanning Mobility Particle Sizer (SMPS: TSI Inc.). The sampling time was in the range between $20 \mathrm{~min}$ and $4 \mathrm{~h}$. The total number of samples taken was 12 for particles of $21 \mathrm{~nm}$ diameter and six for particles of $75 \mathrm{~nm}$ diameter.
The maximum $\mathrm{W}$ mass was found at stage 3 for particles of $21 \mathrm{~nm}$ diameter and at stage 4 for particles of $75 \mathrm{~nm}$ diameter. Thus, the mean value of the aerosol particle size distributions obtained with WRAS was in a very good agreement with the mean size measured obtained with the SMPS. The mass of W obtained with WRAS was compared with the mass calculated from SMPS data. The regression analysis reveals that $R^{2}=0.83$ and the mean ratio of $M_{\mathrm{WRAS}} /$ $M_{\text {Calc }}=1.05$.

It was very difficult to obtain stable aerosols containing particles with diameters $<21 \mathrm{~nm}$ with a photochemical aerosol generator. However, there is no reason to believe that the approach verified for 21 and $75 \mathrm{~nm}$ particles would provide incorrect measurements when sampling smaller particles. The deposition of smaller particles is governed by the same principles as the deposition of particles with 21 and $75 \mathrm{~nm}$ diameter. Moreover, the diffusion deposition model is an approximation that works better for smaller particles than for larger particles. Thus, WRAS instruments can be employed to sample size-selectively aerosol particles in the entire aerosol size range down to nanometre-size particles without using a high-pressure drop. Therefore, sampling artefacts caused by evaporation/condensation of volatile and semi-volatile compounds are minimal for the WRAS instrument.

In addition, the diffusion unit was calibrated in the same way as for a traditional diffusion battery by measuring aerosol particle number concentrations with a condensation particle counter, see Fig. 9. The same photochemical $\mathrm{W}$ generator, as described above, was used: $\sigma_{\mathrm{g}}$ of $\mathrm{W}$ particles was in the range from 1.3 to 1.6. The modal diameter ranges from 6 to $300 \mathrm{~nm}$. On one hand, aerosol particle number concentrations $N p_{i}$ were measured in the flow downstream of the diffusion collector containing only one stage at the time.

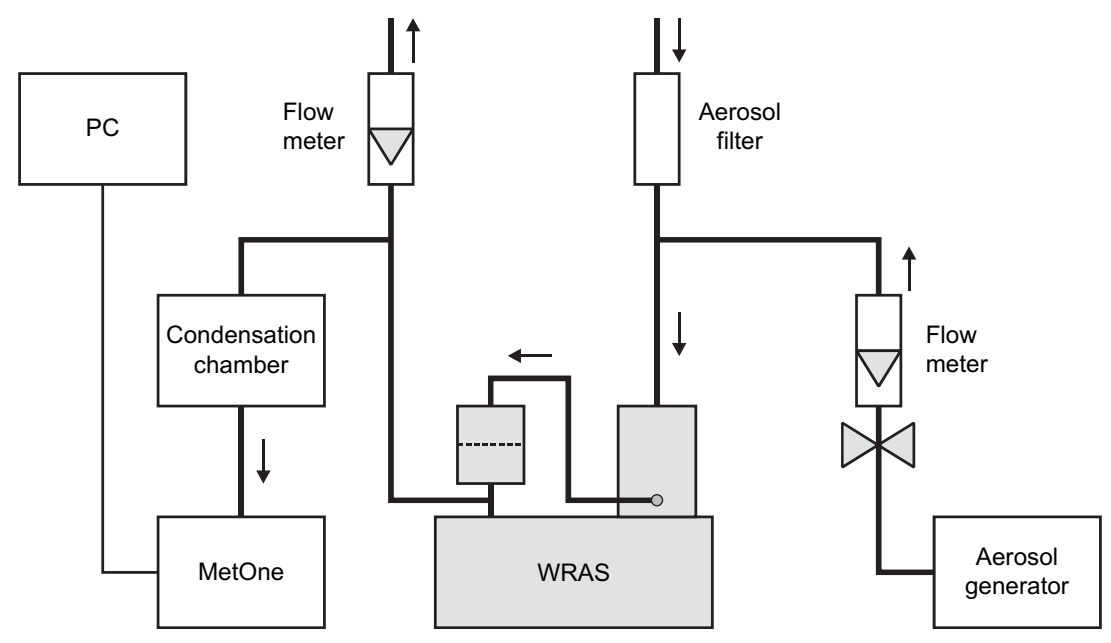

Fig. 9. Schematic of the calibration set up for nanoparticle collector. A dashed line in the nanoparticle collector on the top of WRAS unit shows a single stage of the nanocollector that may have one or several nets. 
On the other hand, aerosol particle number concentrations were calculated according to the deposition theory using the $\mathrm{W}$ aerosol particle size distributions (lognormal approximation) $f(D)$

$$
N p_{i}=N p_{5} \int_{-\infty}^{\infty} P_{i}\left(D, \chi_{i}\right) f(D) \mathrm{d} \log D
$$

Here $N p_{5}$ was the aerosol particle concentration measured downstream of the cascade impactor after passing the fifth stage. The penetration efficiencies $P_{i}\left(D, \chi_{\mathrm{i}}\right)$ in equation (18) were calculated according to the filtration theory with a correction factor $\chi_{1}$ which was expected to be close to 1 if the theory was correct

$$
P_{i}\left(D, \chi_{i}\right)=\exp \left[-\frac{2 N_{i} \alpha_{i} h_{i}}{\pi\left(1-\alpha_{i}\right) a_{i}} \eta_{i}(D) \chi_{i}\right]
$$

Here $N_{i}, \alpha_{i}, a_{i}, h_{i}$ and $\eta_{i}$ are the same parameters as they have been described before (for $i$ th stage). The measured $N_{p i}$ in and the calculated were compared. In total eight different aerosols were generated and used in the comparison. The ratios of $N_{P i}$ in (measured) $/ N_{P i}$ in (calculated) were in the range from 0.75 to 1.3 . Average of $N_{P i}$ in (measured) $/ N_{P i}$ in (calculated) $=1.07 \pm 0.18$. Given that the accuracy of concentration measurements is $\pm 10-15 \%$, this spread shows a sound agreement between the theory and the measurements.

It would be more practical to describe the difference between the theory and the measurements in

Table 3. Calculated and measured maximum cut-off diameters of WRAS diffusion collector stages

\begin{tabular}{lcc}
\hline Stage & Table $2 D_{i}(\mathrm{~nm})$ & Calculated $D_{i}(\mathrm{~nm})$ \\
\hline 2 & 5 & $5.4 \pm 0.7$ \\
3 & 15 & $18 \pm 5$ \\
4 & 60 & $55 \pm 8$ \\
5 & 250 & $240 \pm 20$ \\
\hline
\end{tabular}

terms of the cut-off diameter. A cut-off diameter is one of the main characteristics of the penetration efficiency. Therefore, if the set of cut-off diameters in Table 2 is correct, then calculations of the $N_{P i}$ in based on equations (18) and (19) and experimental data on aerosol size distribution should be close to measured $N_{P i}$ in values. The cut-off diameter $D_{i}$, was calculated from equation (20)

$$
P_{i}\left(D, \chi_{i}\right)=\frac{1}{2}
$$

and compared with $D_{i}$ values from Table 2 . The calculated cut-off diameters were found to be very close to diameters in Table 2, see Table 3.

The first stage correction could not be found due to limitations of the condensation particle counter and difficulties to measure particles with diameter $<0.006 \mu \mathrm{m}$. Thus, the theory used to calculate diffusion deposition onto the stages of the WRAS instrument provides a correct evaluation of the cut-off diameters at $20 \mathrm{dm}^{3} \mathrm{~min}^{-1}$ and, therefore, the theory can be extended and used at higher flow rates or linear velocities up to $19 \mathrm{~cm} \mathrm{~s}^{-1}$.

In the first experiments, a commercial certified May cascade impactor (Scientific Instrument Ltd) was used. The cut-off diameters were supplied by the manufacturer. Later this cascade impactor was replaced by a modified version of the May impactor, where all the stages were positioned horizontally and stage 5 , collecting the smallest particle sizes, was simplified. The simplification was possible because in a WRAS unit there is no need to use an aerosol filter after the final stage of the cascade impactor. This modification enables the price of a unit to be reduced and manufacturing to be simplified.

The modified cascade impactor was manufactured by Naneum Ltd with the aim of having the same set of cut-off diameters as the original May impactor. The number of stages and the main dimensions

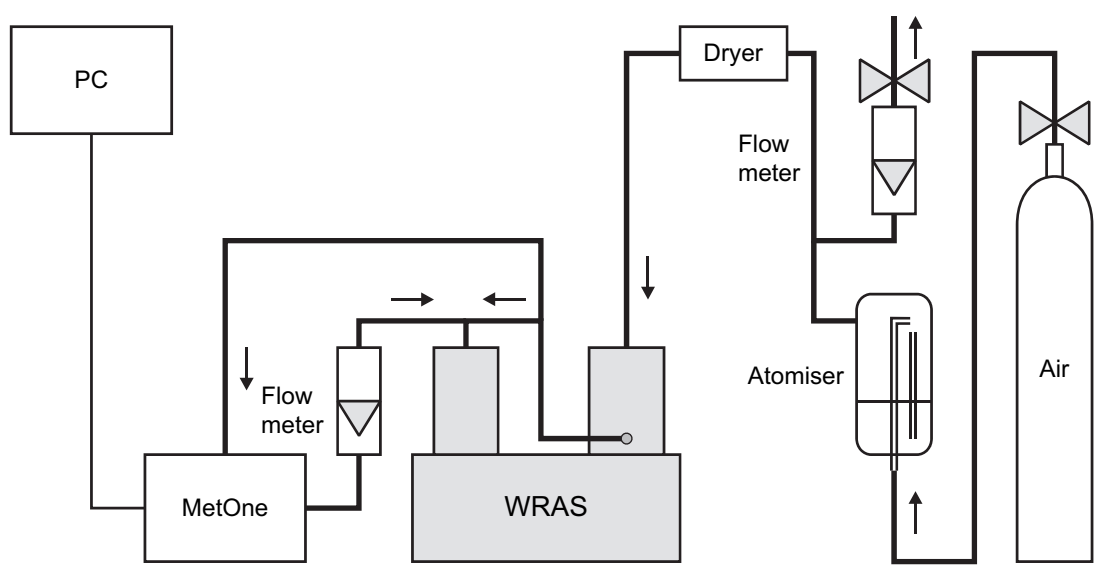

Fig. 10. Schematic of the calibration set up for the modified cascade impactor. 
critical for cut-off diameters were the same in the new impactor as in the original one.

The modified impactor was calibrated using a nebulizer, dryer, MetOne optical particle counter (OPC) and WRAS sampler, Fig. 10. Latex aerosol particles were generated by a nebulizer (Naneum Ltd) from aqueous solutions using a nitrogen cylinder as a source of flow rate of $\sim 30 \mathrm{dm}^{3} \mathrm{~min}^{-1}$. The flow was dried in a dryer operating at $358^{\circ} \mathrm{K}$ and latex particles directed to the inlet of the cascade impactor. In the impactor, several slides could be removed. A MetOne OPC was modified to be able to work inline: the outlet tube of the impactor was extended and placed outside the OPC top cover through an additional orifice.

During calibration of a specific stage, a slide was placed in the impactor in the corresponding slot, e.g. slide in slot 7. All other slides were removed. Deposition efficiency of this stage was measured as a ratio of the number concentration obtained with the slide in and the number concentration obtained when the slide was removed. This measured ratio was compared with the ratio expected according to the aerodynamic diameter of latex particles and the deposition efficiency shown in Fig. 5. The aerodynamic diameter of latex particles was calculated according Fuchs (1964) and Hinds (1999) from the geometric diameter $D_{g}$.

The density of latex particles was assumed to be: $1.05 \mathrm{~kg} \mathrm{~m}^{-3}$. The size of latex particles was chosen to be close to the cut-off diameters of the impactor. For example, for stage 7, two latex monodispense aerosols were used with $D=0.91$ and $1.21 \mu \mathrm{m}$. The actual cut-off was obtained from deposition efficiencies measured with these aerosols by linear regression. For stage 7, it was found to be $1.02 \mu \mathrm{m}$. This is close to the expected value (provided by the May cascade impactor manufacturer) $1.0 \mu \mathrm{m}$.

The calibration was performed in the same way for stage 6 and 9. The difference between the conventional May cascade impactor and the modified one was $<5 \%$ in cut-off diameters. Thus, data provided in Table 2 can be used for both the original May impactor and the new version modified by Naneum Ltd, which is suitable for combination with the diffusion collector.

\section{Examples}

The developed sampling kit (Fig. 11) was modified by Naneum Ltd, UK, incorporating a humiditycontrolling unit (http://www.naneum.com).

Particles were collected at a number of European crystal glass factories (in the UK, Ireland and Sweden) as part of an European Community-funded CRAFT project (BRST-CT97-5122). At these factories, lead aerosols are produced both by the condensation of vapours formed over molten glass and by glass cutting with abrasive disks (WRAS sampler

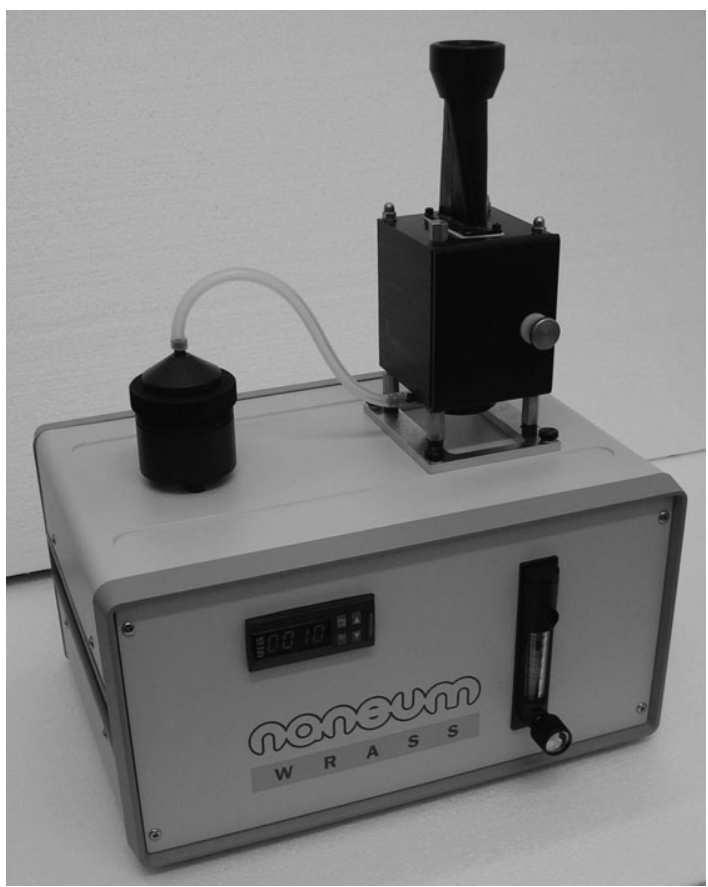

Fig. 11. AWRAS aerosol sampling system with a timer on the left of the front panel, a needle valve to control the flow rate (on the right of the front panel) and a digital flow indicator (on the top).

website, 2006). Aerosol particles were collected in a wide range of sizes from $3 \mathrm{~nm}$ to $20 \mu \mathrm{m}$ (11 size fractions) at a flow rate $20 \mathrm{dm}^{3} \mathrm{~min}^{-1}$ and a relative humidity of $80 \%$.

The sampling time was from 2 to $24 \mathrm{~h}$. The mass of lead collected was determined by AAS with graphite furnace as previously described. Mass concentration size distributions were calculated-Figs 7-9 (The cut-off diameters in Figs 7-9 are different than in Table 2. The instrument is quite flexible as far as choice nets is concerned. Table 2 shows the final recommended set of nets and cut-off diameters. Data in Figs 7-9 were obtained earlier with different sets of nets, therefore, different cut-offs.). The total mass concentration of lead aerosols determined at workplaces ranged from 0.6 to $50 \mu \mathrm{g} \mathrm{m}^{-3}$. The nanoparticle mass fraction of aerosols (sizes $<0.1 \mu \mathrm{m}$ ) was found to vary from 10 to $70 \%$.

\section{CONCLUSIONS}

The principles of inertial and diffusion deposition have been employed in the design and construction of a new instrument (WRAS) that was developed to size selectively collect aerosol particles across a wide aerosol size range — down to nanometre sized particles-for further mass analysis. It was found that for particles $<0.25 \mu \mathrm{m}$, diffusion is the main deposition mechanism for net filters. The instrument 
developed operates at $20 \mathrm{dm}^{3} \mathrm{~min}^{-1}$ and it does not require low pressure to collect nanoparticles. Data calculated using diffusion deposition theory were found to be in a good agreement with experimental data, which indicates that the theory can be extended to higher flow rates (up to $20 \mathrm{dm}^{3} \mathrm{~min}^{-1}$ or $19 \mathrm{~cm}$ $\mathrm{s}^{-1}$ ). Aerosol particle size distributions can be obtained with a simple data reduction technique without the application of complex deconvolution techniques. At working places in factories with high-temperature lead processing, the fraction of lead nanoparticles was found to vary from 10 to $70 \%$.

\section{FUNDING}

Project number BRST-CT97-5122 has been funded by the European Commission.

\section{REFERENCES}

Brown JS, Zeman KL, Bennett WD. (2002) Ultrafine particle deposition and clearance in the healthy and obstructed lung. Am J Respir Crit Care Med; 166: 1240-47.

Busigin A, Van der Vooren AW, Phillips CR. (1980) A technique for calculation of aerosol particle size distributions from indirect measurements. J Aerosol Sci; 11: 359-66.

Chamberlain AC. (1985) Prediction of response of blood lead to airborne and dietary lead from volunteer experiments with lead isotopes. Proc R Soc Lond B Biol Sci; 224: 149-82.

Cheng YS, Yeh HC. (1980) Theory of a screen-type diffusion battery. J Aerosol Sci; 11: 313-20.

Cheng YS, Keating JA, Kanapilly GM. (1980) Theory and calibration of a screen-type diffusion battery. J Aerosol Sci; 11: 549-56.

Cheng YS, Su YF, Newton GJ et al. (1992) Use of a graded diffusion battery in measuring the activity size distribution of thoron progeny. J Aerosol Sci; 23: 361-72.

Dockery DW, Pope CA, Xu X et al. (1993) An association between air pollution and mortality in six US cities. $\mathrm{N}$ Engl $\mathrm{J}$ Med; 329: 1753-59.

Donaldson K, Li XY, MacNee W. (1998) Ultrafine (nanometer) particle-mediated lung injury. J Aerosol Sci; 29: 553-60.

Dubtsov SN, Baklanov AM. (1996) Design and testing of a photochemical aerosol generator for calibrating aerosol measuring instruments in the submicrometer region. Aerosol Sci Technol; 25: 67-72.

Ferin J. (1994) Pulmonary retention and clearance of particles. Toxicol Letters; 72: 121-25.

Ferin J, Oberdorster G, Penny DP. (1992) Pulmonary retention of ultrafine and fine particle in rats. Am J Respir Cell Mol Biol; 6: 535-42.

Fuchs NA. (1964) The mechanics of aerosols. New York, NY: MacMillan Company. pp. 181.

Fuchs NA, Stechkina IB, Starosselskii VI. (1962) On the determination of particle size distribution in polydisperse aerosols by the diffusion method. Br J Appl Phys; 13: 280-81.

Hart KM, Pankow JF. (1994) High-volume air sampler for particle and gas sampling. 2. Use of backup filters to correct for the adsorption of gas-phase polycyclic aromatic hydrocarbons to the front filter. Environ Sci Technol; 28: 655-61.

Hinds WC. (1999) Aerosol technology. Properties, behaviour and measurement of airborne particles. New York, NY: John Wiley and Sons. pp. 233-59.

International commission on radiological protection (ICRP). (1994) Human respiratory tract model for radiological pro- tection. Annals of the ICRP, Publication 66. Tarrytown, NY: Elsevier Science Inc.

Jaques PA, Kim CS. (2000) Measurement of total lung deposition of inhaled ultrafine particles in healthy men and women. Inhal Toxicol; 12: 715-31.

John W. (2001) Size distribution characteristics of aerosols. In: Baron PA, Willeke K, editors. Aerosol measurement: principles, techniques, and applications. New York, NY: John Wiley and Sons. pp. 99-116.

Kirsh AA, Stechkina IB. (1978) The theory of aerosol filtration with fibrous filters. In: Shaw DT, editor. Fundamentals of aerosol science. New York, NY: Wiley. pp. 165-256.

Lee KW, Connick PA, Gieseke JA. (1981) Extension of the screen type diffusion battery theory. J Aerosol Sci; 12: 385-6.

Lesnic D, Elliott L, Ingham DB. (1995) An inversion method for the determination of the particle size distribution from diffusion battery measurements. J Aerosol Sci; 26: 797-812.

Lippmann M. (1995) Size-selective health hazard sampling. Air sampling instruments for evaluation of atmospheric contaminants. 8th edn. Cincinnati, $\mathrm{OH}$ : American Conference of Governmental Industrial Hygienists.

Maher EF, Laird NM. (1985) EM algorithm reconstruction of particle size distributions from diffusion battery data. J Aerosol Sci; 16: 557-70.

Marple VA, Olson BA, Rubow KL. (2001) Inertial, gravitational, centrifugal and thermal collection techniques. In: Baron PA and Willeke K, editors. Aerosol measurement: principles, techniques, and applications. New York, NY: Wiley.

Mavliev R, Ankilov A, Baklanov A et al. (1984) Development and use of a screen diffusion battery for determining aerosol size distribution. Colloid J; 46: 1136-41.

May KR. (1945) The cascade impactor: an instrument for sampling coarse aerosols. J Sci Instrum; 22: 187.

May KR. (1982) A personal note on the history of the cascade impactor. J Aerosol Sci; 13: 37-47.

Mercer TT, Greene TD. (1974) Interpretation of diffusion battery data. J Aerosol Sci; 5: 251-5.

Moore M, Gorbunov B, Williams I. (1998) A new method to study interaction of semi-volatile compounds with aerosol particles. J Aerosol Sci; 29 (Suppl. 1): S887-8.

Reineking A, Porstendorfer J. (1990) Unattached fraction of short-lived $\mathrm{Rn}$ decay products in indoor and outdoor environments-an improved single-screen method and results. Health Phys; 58: 715-27.

Schiller CF, Gebhart J, Heyder J et al. (1988) Deposition of monodisperse insoluble aerosol particles in the 0.005 to $0.2 \mu \mathrm{m}$ size range within the human respiratory tract. Ann Occup Hyg; 32 (Suppl 1): 41-9.

Sinclair D. (1972) A portable diffusion battery. Am Ind Hyg Assoc J; 33: 729-35.

Sinclair D, Countess RJ, Liu BYH et al. (1976) Experimental verification of diffusion battery theory. J Air Pollut Control Assoc; 26: 661-3.

Sinclair D, Hoopes GS. (1975) A novel form of diffusion battery. Am Ind Hyg Assoc J; 36: 39-42.

Soderholm SC. (1979) Analysis of diffusion battery data. J Aerosol Sci; 10: 163-75.

Thomas JW. (1955) The diffusion battery method for aerosol particle size determination. J Colloid Sci; 10: 246.

Twomey S. (1975) Comparison of constrained linear inversion and an iterative non-linear algorithm applied to the indirect estimation of particle size distributions. J Comput Phys; 13: 188-200.

Wilson FJ Jr, Hiller FC, Wilson JD et al. (1985) Quantitative deposition of ultrafine stable particles in the human respiratory tract. J Appl Physiol; 58: 223-9.

Yeh HC, Cuddihy RG, Phalen RF et al. (1996) Comparisons of calculated respiratory tract deposition of particles based on the proposed NCRP model and the new ICRP model. Aerosol Sci Technol; 25: 134-40.

WRAS sampler website. (2006) Available at http://www. naneum.com. Accessed January 2009. 